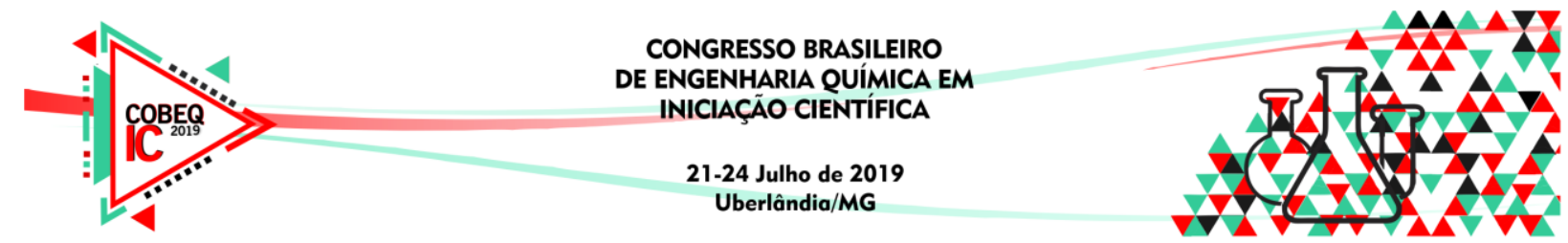

\title{
APLICAÇÃO DE SUPPORT VECTOR MACHINE NA DETECÇÃO DE FALHAS EM SENSORES DE UMA COLUNA DEBUTANIZADORA
}

\author{
L. C. BARRETO ${ }^{1}$, B. C. V. SANTOS ${ }^{1}$, A. P. M. R. SOARES ${ }^{1}$, G. D. SILVA $^{1}$ e \\ F. O. CARVALHO ${ }^{1}$ \\ ${ }^{1}$ Universidade Federal de Alagoas, Centro de Tecnologia \\ E-mail para contato: lucascosta_108@hotmail.com
}

\begin{abstract}
RESUMO - Com a automatização de processos tecnológicos dentro do ambiente industrial, atrelada a presença de sistemas de controle e monitoramento das plantas, faz-se necessário a garantia da integridade das informações enviadas a rede de controle e monitoramento, para que ela possa operar sem falhas que comprometam a produção. Visando atender a essa necessidade, esse trabalho propôs a utilização da técnica de inteligência artificial Máquina de Vetores Suporte (do inglês Support Vector Machine, (SVM)), para criação de um sistema de detecção de falhas em sensores industriais. $\mathrm{O}$ estudo de caso foi realizado em sensores instalados em uma coluna debutanizadora que opera em uma planta de dessulfurização. O modelo se mostrou satisfatório na detecção das falhas nos sensores. Os resultados mostraram que a Support Vector Machine obteve desempenho satisfatório na detecção de falhas para este tipo de problema.
\end{abstract}

\section{INTRODUÇÃO}

Segundo Chiang et al. (2001), o aumento da procura por sistemas de detecção de falhas é justificado pelo fato de os processos industriais estarem se tornando cada vez mais instrumentados, o que resulta em uma grande quantidade de dados disponíveis para utilização no monitoramento. Estes dados ficam armazenados em computadores robustos, sob a forma de históricos do processo, e são extremamente necessários para análise da operação. Isto levou ao desenvolvimento de ferramentas de detecção e diagnóstico de falhas baseadas na análise destes históricos, eliminando a necessidade de se utilizar modelagem fenomenológica do processo. Dentre estas ferramentas, pode-se citar a support vector machine (SVM), que foi utilizada neste trabalho.

Devido ao seu forte respaldo matemático, tendo construção mais geral e aplicabilidade mais ampla, e por ter apresentado alto desempenho em aplicações práticas, inclusive superior a muitos algoritmos de aprendizagem conhecidos, as SVMs têm atraído o interesse da comunidade de aprendizagem de máquina (SOUZA, 2011).

Sendo assim, esse trabalho buscou investigar a aplicação das SVM, técnica baseada em inteligência artificial oriunda do conceito estatístico, para o problema de detecção de falhas em sensores. Para tal, foram utilizados registros de sensores de uma coluna debutanizadora, obtidos em Fortuna et al. (2007). 


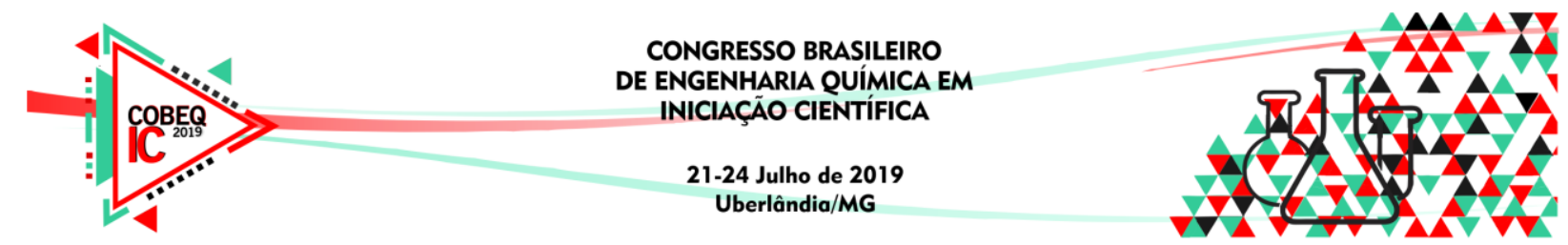

\subsection{Máquinas de Vetores Suporte}

As máquinas de vetor de suporte (ou SVMs, do inglês support vector machines), propostas inicialmente por Vapnik (1999), consistem em uma categoria de aprendizado de máquina que tem fundamentação teórica na teoria de aprendizagem estatística desenvolvida por Vapinik (1999) e podem ser utilizadas na classificação de conjunto de dados linearmente e não linearmente separáveis, especificamente, no caso desse trabalho na detecção de falhas, como reportado por Silva (2012) na detecção de falhas em motores elétricos e por Granzotto e Oliveira-Lopes (2015) na detecção de falhas em um sistema para a produção de ciclopentenol em um CSTR.

O fundamento básico do funcionamento de uma support vector machine é a construção de um hiperplano (objeto matemático), o qual pode possuir uma dimensão $\mathrm{R}^{\mathrm{n}}$, possibilitando a separação dos dados em classes distintas. Essa separação tem abordagem baseada na Teoria de Aprendizagem Estatística, utilizando a otimização matemática, para promover a busca de um hiperplano ótimo, adotando os mínimos quadrados por meio da minimização estrutural de risco. A otimização é realizada por meio de programação quadrática ou convexa, baseadas no método dos multiplicadores de Lagrange (SARAIVA, 2017).

A equação geral de um hiperplano é dada pela equação 1 , onde $w$ é o vetor peso e $b$, o bias, que são ajustados de acordo com os dados fornecidos na etapa de treinamento $(x)$, procedimento que se repete até que o hiperplano seja capaz de executar a separação dos dados em diferentes classes (BEN-HUR, WESTON, 2010).

$$
f(x)=w \cdot x+b
$$

Para a resolução de problemas de natureza não linear, é utilizado um artifício matemático de transformação de espaço vetorial chamado de uma função kernel. Desta forma, essas funções são utilizadas na SVM para realizar o mapeamento e a mudança do espaço característico do problema para um de maior dimensão, em que os dados possam ser linearmente separáveis (SARAIVA, 2017). Os tipos de função kernel mais utilizadas são: Linear, Polinomial e Gaussiana (RBF), dadas pelas equações 2,3 e 4, respectivamente.

$$
\begin{aligned}
& K=x_{j}^{T} x_{j} \\
& K=\left(x_{i}^{T} x_{j}-k\right)^{p} \\
& K=\exp \left(-\frac{\left\|x_{i}-x_{j}\right\|^{2}}{2 o^{2}}\right)
\end{aligned}
$$

Para o kernel Polinomial (Equação 3), $p$ e $k$ devem ser especificadas e para o Gaussiano (Equação 4), a amplitude $o^{2}$ deve ser especificada.

\section{METODOLOGIA}

\subsection{Descrição do Conjunto de Dados}




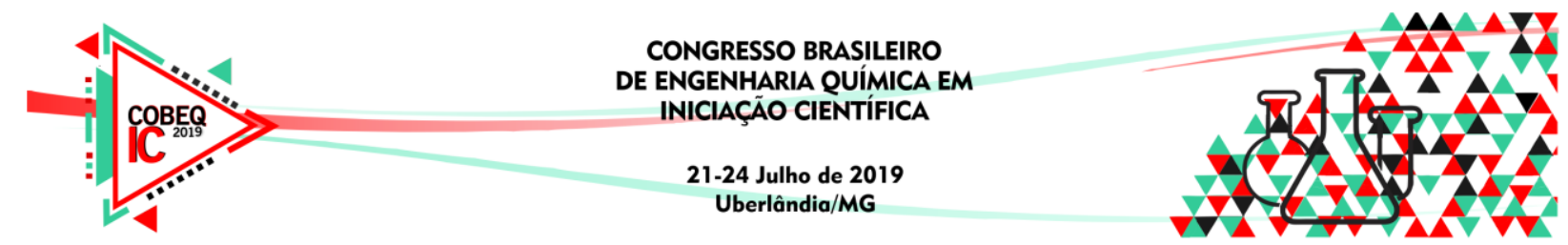

A coluna debutanizadora em estudo opera em uma planta de dessulfurização e separação de nafta, a Figura 1 mostra o esquema da coluna e as variáveis envolvidas nessa etapa do processo.

Figura 1 - Esquema de uma coluna debutanizadora e variáveis inerentes ao processo.

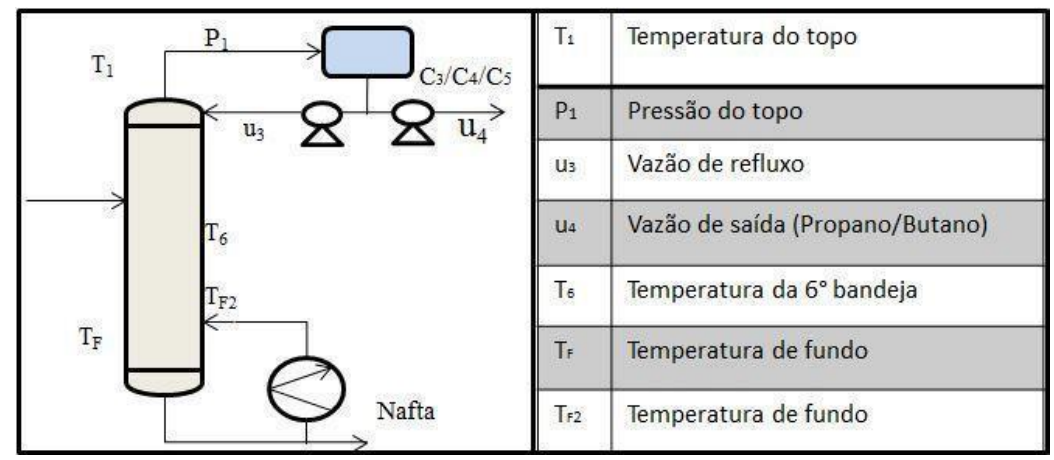

Devido a frequente ocorrência de falhas em sensores de temperatura e pressão, estes foram considerados para o estudo de caso. No total, o conjunto de dados continha 2394 registros referentes às medições contínuas realizadas no equipamento dentro de um determinado intervalo de tempo. Todo esse conjunto foi obtido em Fortuna et al. (2007), vale salientar que os dados disponibilizados na literatura citada já estavam normalizados e tratados estatisticamente e prontos para utilização para construção do modelo de detecção de falhas.

\subsection{Simulação das Falhas e Construção dos Modelos}

Foram inseridas, aleatoriamente, falhas nas medições dos sensores de temperatura de topo e pressão de topo dentro de um intervalo de $\pm 30 \%$ em relação ao valor real. Após essa etapa, o conjunto total dos dados (com e sem falha) passou a ter 7182 registros, os quais poderiam ser classificados dentro dos seguintes grupos:

- Grupo 1: Falha no sensor de pressão de topo;

- Grupo 2: Falha no sensor de temperatura de topo;

- Grupo 3: Falha simultânea dos sensores;

- Grupo 4: Funcionamento normal do sistema.

\subsection{Treinamento, Validação e Topologia dos Modelos}

O modelo desenvolvido com a utilização das Máquinas de Vetores suporte, foi treinado apenas para identificar a ocorrência de falhas nos sensores, sem necessariamente discernir qual dos sensores apresentava a falha, o que seria o diagnóstico. Isso ocorreu em razão das limitações computacionais encontradas no software utilizado, para essa ferramenta em específico. Dessa forma, os grupos 1, 2 e 3 se tornaram uma só classe e o grupo 4 uma outra classe. Sendo assim, o problema passou a ter apenas duas classes (dados com ou sem falha) sendo a classe 0 relativa aos dados sem a inserção de falhas e a classe 1 (mostradas na matriz de confusão Figura 2) relativa aos dados que representavam a operação da coluna com falhas operacionais. 


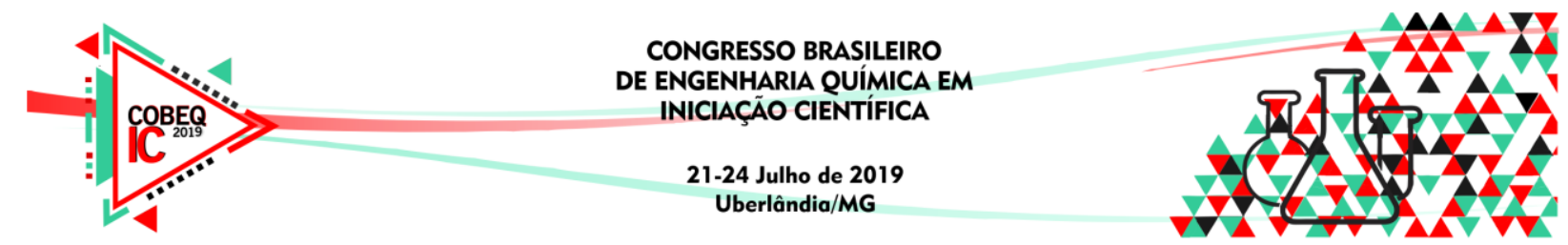

Vale salientar que a escolha da função kernel do modelo SVM foi selecionada através de métodos empíricos. Desta forma, a função kernel Gaussiana (RBF) foi a que apresentou melhor desempenho para o conjunto de dados analisados neste trabalho.

Todo o sistema de detecção de falhas, desde a simulação das falhas e treinamento dos modelos, foi desenvolvido utilizando a linguagem de programação Python®. Vale salientar que na etapa de validação do modelo, foram utilizados $15 \%$ dos dados do conjunto original, registros esses que não foram utilizados na etapa de treino. Desta forma, foi possível avaliar a capacidade de generalização do modelo desenvolvido.

\section{RESULTADOS}

A avaliação do modelo desenvolvidos foi realizada a partir da observação dos resultados de desempenho mostrados na matriz de confusão geradas na etapa de validação (Figura 2).

Figura 2 - Matriz de Confusão para o sistema de detecção de falhas com o modelo Máquinas de Vetores Suporte (SVM).

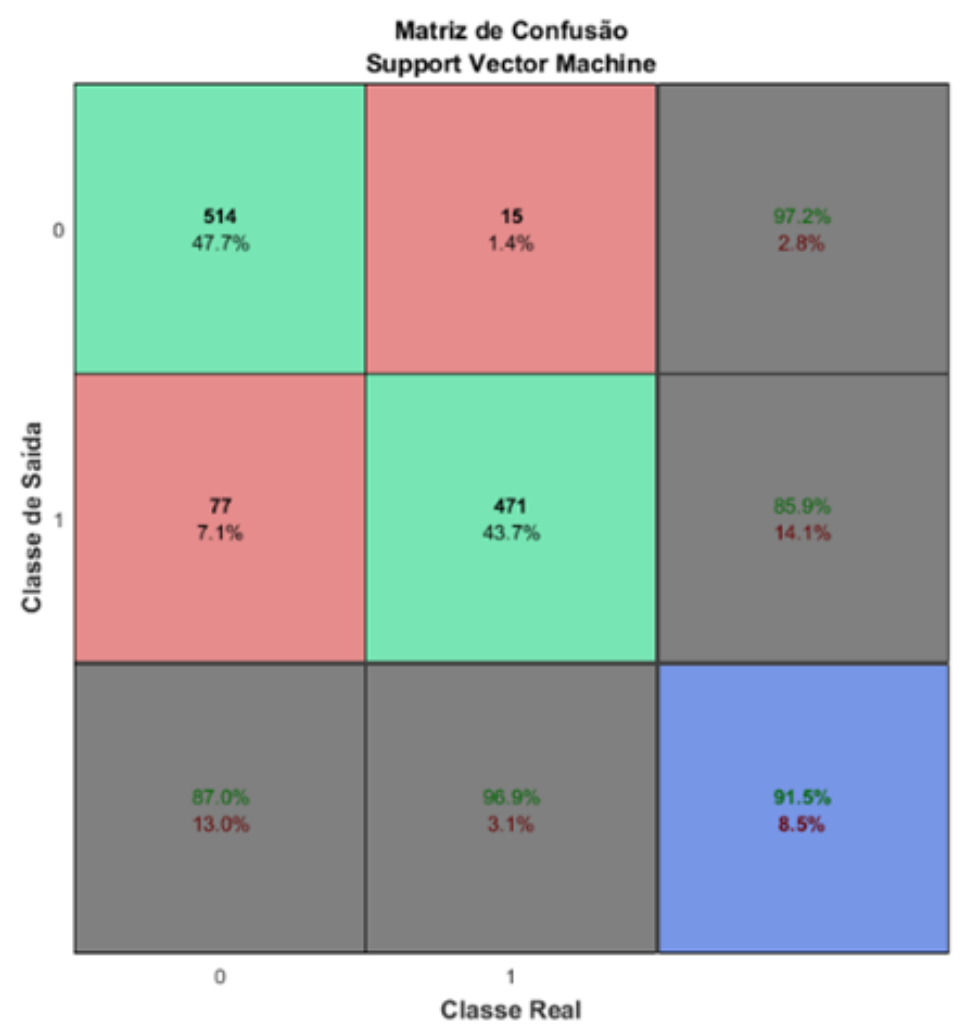

A partir da Figura 2 (Modelo Máquinas de Vetores Suporte) é possível ver o percentual de acertos na classificação do modelo na fase de validação. Desta forma, podemos perceber que dos 529 casos de teste para a classe 1, que representa o perfeito funcionamento do sistema, 514 $(97,2 \%)$ foram classificados corretamente. A classe 2 por sua vez, foi identificada corretamente 471 vezes $(85,9 \%)$, dos 548 casos testados. Dessa forma, o modelo obteve uma taxa de acerto global de 91,5\%, desempenho que denota a eficiência e aplicabilidade do modelo de classificação SVM para o estudo de caso avaliado neste trabalho. 


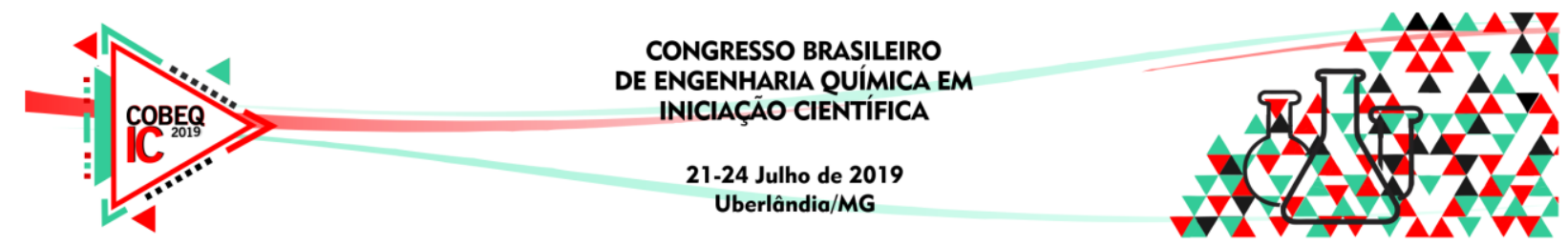

\section{CONCLUSÃO}

Neste trabalho foi demonstrada a construção de um sistema de detecção de falhas em sensores industriais. A análise dos resultados obtidos nos permite dizer que o modelo de detecção de falhas desenvolvido neste trabalho a partir da técnica de classificação com a Máquinas de Vetores Suporte teve uma alta taxa de acerto, além disso, poucos foram os registros de alarmes falsos e erros de classificação por parte dessa técnica. Dessa forma, podemos concluir que a SVM se mostrou uma técnica bastante promissora no que diz respeito a sua utilização na rede de comunicação industrial. Sendo assim, os resultados obtidos neste trabalho demonstram que o modelo desenvolvido tem potencial de utilização para o estudo de caso analisado. Entretanto, para trabalhos posteriores pensa-se no desenvolvimento de um modelo que execute também o diagnóstico das falhas, de modo que seja possível não somente identifica-las como também saber o equipamento ou sensor que deu origem a essa falha.

\section{REFERÊNCIAS}

CHIANG, L. H.; RUSSELL, E. L.; BRAATZ, R. D. Fault Detection and Diagnosis in Industrial Systems. Londres: Springer, 2001.

BEM-HUR, A.; WESTSON J. A User's guide to Support Vector Machine data maning techniques for the Life Sciences. Methods in Molecular biology, v. 609, 2010.

FORTUNA, L.; GRAZIANI, S.; RIZZO, A.; XIBILIA, M. G. Soft Sensors for Monitoring and Control of Industrial Processes. Londres: Springer, 2007.

GRANZOTTO, M. H.; OLIVEIRA-LOPES, L. C.; "Desenvolvimento de Sistema de Detecção de Falhas Baseado em Aprendizado Estatístico de Máquinas de Vetores de Suporte", p. 11819-11828. In: Anais do XX Congresso Brasileiro de Engenharia Química - COBEQ 2014 [ = Blucher Chemical Engineering Proceedings, v.1, n.2]. São Paulo: Blucher, 2015.

SARAIVA, S. V. Estudo de um controlador preditivo baseado em sistemas inteligentes. Trabalho de conclusão de curso. Universidade Federal de Alagoas, Maceió, 2017.

SILVA, V. A. D. Detecção de falhas em motores elétricos através das máquinas de vetores de suporte. Dissertação (Mestrado) - Universidade Estadual de Campinas, Faculdade de Engenharia Mecânica, Campinas, 2012. Disponível em:

<http://www.repositorio.unicamp.br/handle/REPOSIP/265564>. Acesso em: 13 mar. 2019.

SOUZA, F. C. M. Técnicas de busca heurística para otimização de parâmetros de Máquinas de Vetores Suportes. Dissertação submetida ao programa de Pós-Graduação em Ciência da Computação. Universidade Federal de Pernambuco, Recife, 2011.

VAPNIK, V. N. The Nature of Statistical Learning Theory. Springer Verlag, New York, $2^{\text {nd }}$ edition, 1999. 